\title{
DiffServ in the Web: \\ Different Approaches for Enabling Better Services in the World Wide Web
}

\author{
Hartmut Ritter, Thorsten Pastoors, and Klaus Wehrle \\ Institute of Telematics, University of Karlsruhe (TH), \\ D-76128 Karlsruhe, Germany \\ \{ritter, pastoors, wehrle\}@telematik.informatik.uni-karlsruhe.de
}

\begin{abstract}
The paper discusses several approaches to use the Differentiated Services Architecture for providing better services to the users of the World Wide Web. In the future Internet a user wants to have the possibility to select between different service levels to get specific web-contents. The sender orientation of the Differentiated Services Architecture imposes some difficulties in providing the user with a means for determining the service he wants to get out of a web-server. In this paper three approaches for providing better services to the users of the World Wide Web based on the Differentiated Services Architecture are presented. Additionally, an overview over implementation issues using Linux is presented.
\end{abstract}

\section{Introduction}

Along with the enormous growth of the Internet the demands for network services have become increasingly higher. Among other things, services are required that assure certain quality of service parameters, e.g. guaranteed bandwidth, maximum packet delay or minimum variations in the inter-arrival time of consecutive packets (jitter). The World Wide Web, which can be seen as the most important and most used service in the Internet, offers only best effort service. Downloading huge files sometimes takes endlessly, because of too many packet losses resulting in long delays. A service better than best effort is needed. Providers of web services also would like to offer alternative levels of service to their customers in order to charge for higher quality. The actually most discussed approach to provide alternative service levels in the Internet is the Differentiated Services Architecture ([1]). Section 2 gives a brief introduction into the Differentiated Services Architecture.

Providing better services to the World Wide Web would mean, that the webserver responds to an HTTP request using a service better than best effort for some or all of the requests. This can be done statically, i.e. the web-server always sends using better service or distinguishes according to the source of the HTTP request or according to the requested content. This might be interesting for ISPs 
which could give, for example, their clients a better access to a search engine. Nevertheless, in the common case, using the World Wide Web means to move frequently from one server to another. A normal web user does not want to use only a few good servers, but sometimes wants the possibility to get a better service of the web-server he actually visits.

The focus of this paper therefore is, how to give the user the possibility to express his actual request for a better service. The Differentiated Services Architecture, as being sender oriented, imposes some difficulties here. In a clientserver scenario, typical in the World Wide Web, the request issued by the client (the web-browser) is rather unimportant with respect to quality features. The overall quality of service is determined in the first place by the service level of the response. Thus, there should be a way for the user on the client side to influence the service level of the response.

In section 3 three different approaches to overcome the problem of sender orientation are discussed. Section 4 describes the implementation under the Linux operating system. Section [5 finally presents a summary and discusses further issues. Especially the problem of pricing has to be discussed when deploying service differentiation in the Internet of the future.

\section{Differentiated Services}

The mechanisms of the Differentiated Services Architecture (DS Architecture) are held consciously simple in order to guarantee the scalability within network nodes. The architecture distinguishes three different router models.

At the boundary of the first domain acts the so-called ingress boundary node, normally the first router of the data path. Its task is the distinction of all individual traffic streams and their inspection with regard to the traffic conditioning agreement (TCA). If the TCA is exceeded, the boundary node has to form the traffic corresponding to the reservation in the TCA. In order to protect the subsequent intermediate systems from further fine granular classification the ingress boundary node records the classified service in the IP packet header by setting a codepoint in the Differentiated Services field (DS field, the former TOS field).

Normally, the ingress boundary node is the only instance that can select the service class by setting a codepoint. But the Differentiated Services Architecture [1] takes also into account that the sender can already choose the codepoint for each packet. This method is called pre-marking. The subsequent ingress boundary node will additionally police the marked packets in reference to the TCA. If a packet conforms to the TCA it can pass the router with the pre-marked codepoint, otherwise the packet would suffer a degradation of the service (e.g. marking as Best-Effort, discarding, etc.). Pre-Marking allows the sender - a web-server for instance - to choose the service for a flow. Therefore, some kind of reservation has to be established previously. Section 2.1] will take a closer look at reservations and management issues.

The routers of the further communication path only consider the entire aggregate of all flows of a service by looking at the DS field in the IP packet header. 
In the interior of a domain, all packets are only influenced by simple queuing mechanisms, e.g. several queues - traffic shaping and policing are not carried out.

\subsection{Management of Differentiated Services}

As mentioned in 2], at least for Premium Service admission control and resource reservation are required. Furthermore, installation and updating of traffic profiles in boundary nodes are necessary. Most network administrators will not accomplish this task manually, even for long term service level agreements. Furthermore, offering services on demand requires some kind of bandwidth request messages and automatic admission control procedures. Therefore, the concept of Bandwidth Brokers was already suggested by Van Jacobson at a very early stage of Differentiated Services research [3]. In this concept, the Bandwidth Bro$\operatorname{ker}(B B)$ is a dedicated node in each DS domain, keeping track of the amount of available and reserved bandwidth for services, and processing admission control requests from customers or Bandwidth Brokers of adjacent domains. Additionally, it also installs or alters traffic profiles in boundary nodes.

In this paper it is assumed that a Bandwidth Broker allows to negotiate parameters like the sending rate for aggregated flows between the web-server and the web-client. The Bandwidth Broker or the corresponding ISP may also perform accounting in order to enable usage-based pricing.

\section{Approaches for Enabling Differentiated Services in the WWW}

With the way Differentiated Services are defined today, network traffic from a sender to a receiver can only be pre-marked and shaped on the sender's initiative. But often, this is not what is wanted. As stated earlier, in a typical World Wide Web scenario the user issues a short, rather unimportant (with respect to quality of service) request and awaits an important reply. Traffic from the web-server to the client - the reply - can only be pre-marked and shaped on the server's initiative. The problem that arises is obvious: How does the server know what reply is important for the client, and therefore needs a certain quality of service?

As this question deals with the client's desires, the best solution would be to let the client decide which service to use for the reply. It can most of the time better decide which quality, and hence service, would be appropriate. The main problem is therefore, how to pass the client's desires to the server. The solution should be as simple as possible, in order to avoid basic changes in the Differentiated Services Architecture, as well as in the existing client-server applications. The deployment of a dedicated signaling protocol creating overhead is also considered not to be appropriate.

Setting the codepoint used in the servers response is sufficient for changing the service level. But additionally, considering Premium Service, e.g., the specification of a rate is useful. If the aggregated rate of Premium Service traffic in 
the first boundary node grows above a specified limit, packets may be delayed or discarded. Therefore, the web-server should also use an implementation of Differentiated Services that performs traffic shaping and traffic control in the same manner as in the first boundary node. In this paper, it is assumed that some kind of Bandwidth Broker provides the web-server with the information about the specified limits, e.g. the maximum rate of Premium Service flows.

In 2] some measurements have been presented, that show that the TCP protocol has extensive problems using the total amount of a reserved bandwidth. In some of the examples presented in 2] the average rate of a TCP connection over the entire transmission time was only about $20 \%$ of the reserved bandwidth. This problem can be solved in the sender with a strict traffic shaping of an outgoing TCP stream. The shaper takes care that the reserved traffic rate is not exceeded and thus, no packets will be discarded in the Differentiated Services routers.

Nevertheless, the user on the client side might not wish the server to send with the maximum rate but with a rate adapted to the local conditions. A client connected via a small-bandwidth dial-up link is constrained to the link bandwidth and does not take any advantage from a faster web-server; depending on the reservation scheme a huge percentage of Premium Service packets will be discarded at the egress node in order to avoid starvation of best-effort traffic. Considering Assured Service, the situation is similar. Thus, a second question is to be answered: How does the server know what quality of service parameters the client needs?

The following describes three different approaches that try to solve these problems under the given restrictions. For each approach its advantages as well as its disadvantages are discussed. Section 4 describes an implementation for two of the presented approaches.

\subsection{Service Differentiation via Separate HTML Links}

In the first approach, as shown in figure 1 the provider of the web-content provides several links for a specific web-content. Each link allows for downloading this web-content with a different performance when following the link. For example, a company offers five links for downloading a fifty megabyte update of its software. Figure 2 shows an example as the client would see it in his webbrowser. The first link offers downloading with best effort and the next four links offer downloading with better services - whether with Premium Service or with Assured Service.

The advantage of this approach is that the user only has to distinguish between the different possibilities given in the web-page. There is no need for modifying the browser or integrating a plug-in. Each of these links (except for the first one) carries several parameters describing the service, as e.g. service level, rate, burst size, fee, etc. As soon as the customer chooses one of the links with a better service, an HTTP Get command is sent to the web-server - including the quality of service parameters for downloading the data. Encoding the rate and burst-size in the link of a web page would then look like the following: 


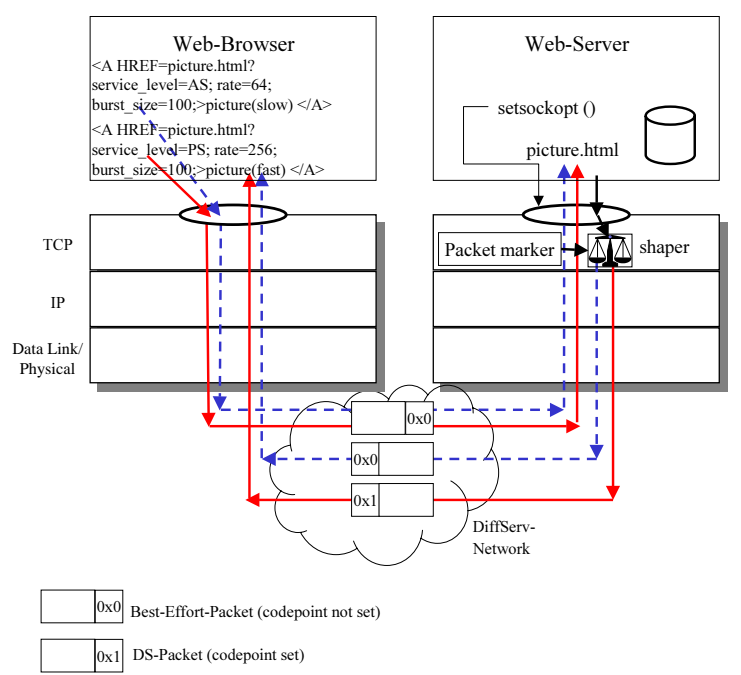

Fig. 1. Service Differentiation via Separate HTML Links

http://www.foo.com/index.html?service=PS; rate=64; burst_size=100; This method of passing additional parameters is conform to the way an HTTP URL scheme is defined in [4].

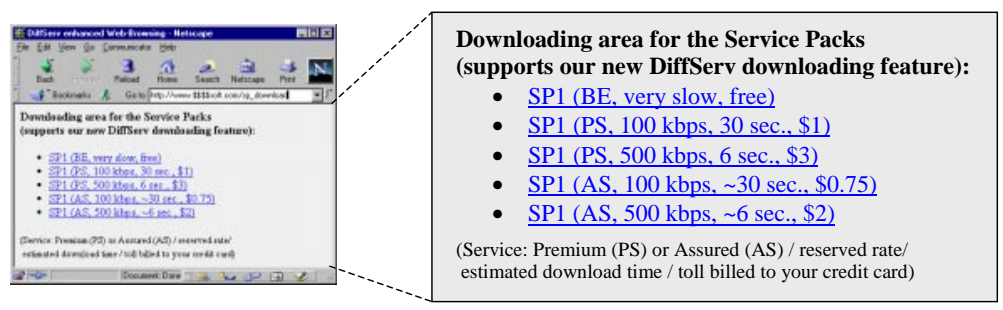

Fig. 2. Example of a Web Site offering Service Differentiation via Separate HTML Links

The web-server parses the get requests and extracts these parameters. Next, it contacts the Bandwidth Broker in order to negotiate the admitted rate and sends the data to the client. Finally, the web-server issues a system call to the TCP socket in order to set the codepoint of the outgoing packets of this connection (pre-marking). Additionally, it passes the quality of service parameters to the network subsystem. Using these parameters the network subsystem performs traffic shaping for all flows better than best-effort.

Because the service selection is coded in the HTTP request and these parameters must be passed down to the network subsystem, this approach requires changes both to the web-server application and the network subsystem of the 
web-server. This can be seen as a disadvantage; on the other hand this enables the client system to remain totally unchanged. The modifications of the Apache web-server turned out to be minor patches (cf. section 4). Another disadvantage, however, might be that the web-pages need modifications. Multiplying all links on a web page would lead to a bad usability. Thus, this approach fits best for download areas as in the given example.

\subsection{Service Differentiation via HTTP Tags}

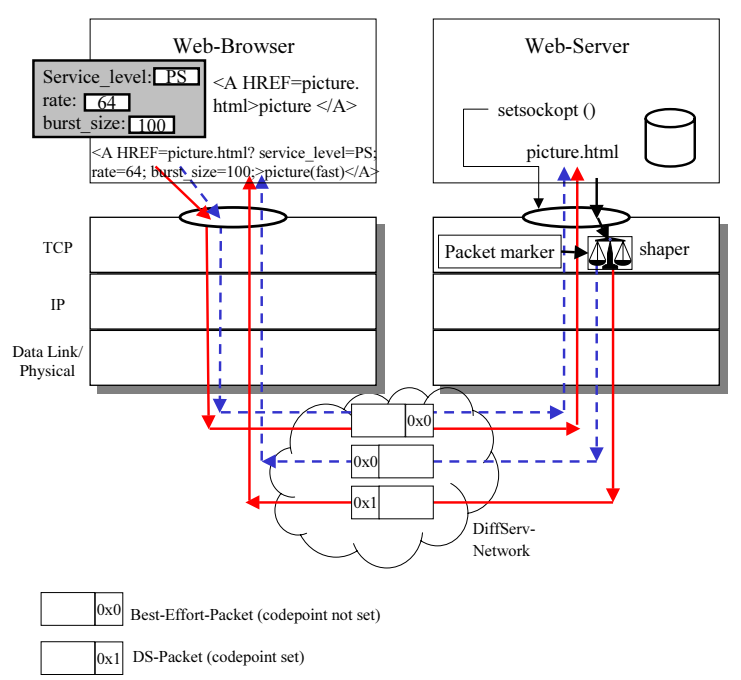

Fig. 3. Service Differentiation via HTML Tags

The second approach, shown in figure 3, is similar to the previous one. However, the way the user chooses the quality of a specific web-content differs. Using this approach, the web-page only provides a single link for a specific web-content as it usually does today. With a click on the link with the left mouse button, the content is transferred as it is today - with best effort. On a click with the right button a menu pops up, with an additional entry for getting this web-content with some better service. Choosing this entry pops up another window in which the user specifies the rate and burst-length with which he wants to receive the content. From there on, this approach behaves like the previous one. An HTTP Get command is sent to the web-server - including the parameters specified by the user. The web-server negotiates the parameters with the Bandwidth Broker and sends the data to the client as described above.

The main difference in this method compared to the previous one is the fact, that here, the web-client needs to be modified: The additional field in the popup window, as well as the window for submitting rate and burstlength have to 
be integrated. The web-pages however, could be the ones we have today. Thus, doubling the links can be avoided. This is obviously an advantage. Furthermore, this approach is also more flexible with respect to choosing the quality of service parameters. On the other hand, this might be a very demanding task for normal users, as a deeper understanding of the parameters would be necessary. A user who is not informed about its actual link bandwidth will have problems to choose the appropriate values.

\subsection{Service Differentiation via Packet Marking}

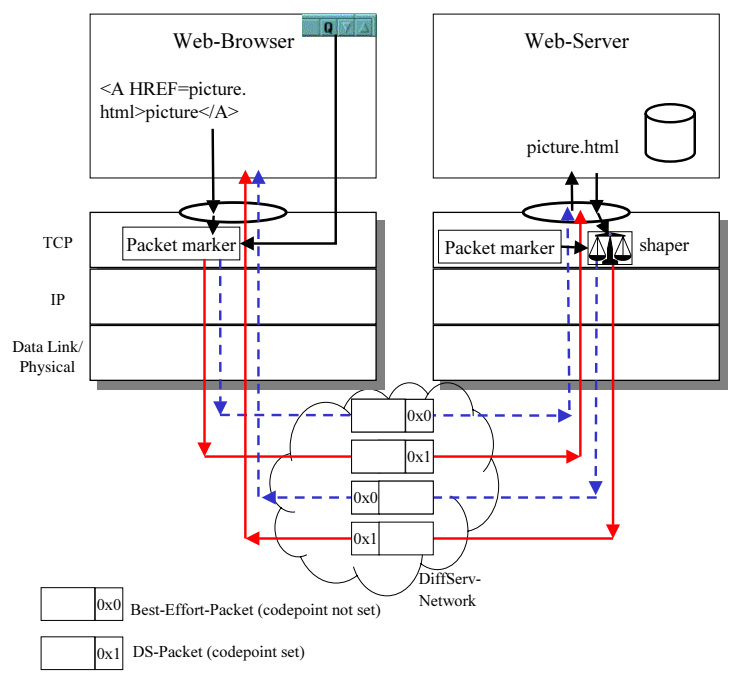

Fig. 4. Service Differentiation via Packet Marking

In this approach the server system sets the codepoint in the DS field to the appropriate value according to the codepoint of the IP-packets of the client request. The codepoint of the client request is set by the user of the web-browser. As stated before, this pre-marking of packets is described in the Differentiated Services Architecture. Due to the small bandwidth required by an HTTP request, the request is very likely to be passed across the network to the web-browser. The server system, where the web-server is running on, detects incoming packets with the codepoint set. This codepoint is then copied to all packets sent by the server on this TCP connection. By this simple means, the client system can determine the service level of the response of the web-server.

To express his current preferences, the user can not use different HTML-links on the web site. Instead, the user needs another interface for expressing his or her current preferences. One approach for a simple interface, the Q-Button, is presented in [5]. In the corner of the web-browser window an additional button is integrated: This button is labeled with the letter Q, denoting Quality of 
Service. It is not part of the application, but of the window manager. The user expresses the current preference for an application running in the related window by clicking on the Q-Button in the window bar. If the user presses the button, this triggers a packet marker in the kernel to mark outgoing packets of the TCP connections of this application.

The advantage of this approach is its simplicity: The simple interaction of pressing a button results in a better service. A service degradation to best effort can be achieved by right-clicking the button. Changes to the web-pages or to the web-browser as in the other two approaches are not necessary. The webserver application also remains unchanged, as the packet marking is done in the network subsystem of the server. Thus, the approach requires no changes to the applications, but the integration of a packet marker into the network subsystem, which can be done quite easily, at least under Linux. The approach fits best for dynamic changes of the service level. Requesting a better service neither requires another parameter menu nor must the web-page support this explicitly.

The answer to the second question ("How does the server know what quality of service parameter the client needs?") however is more problematic. The concept does not allow passing a set of technical parameters like the desired rate to the server. Explicit signaling for every short-term connection is very likely to create too much overhead. Depending on the future devlopment of the Differentiated Services Architecture some feedback to the web-server might be possible, be it by simple backward congestion indication or be it by a notice from the Bandwidth Broker. In the mean time, this approach seems to fit best for short interactions, typical in many World Wide Web sessions. The download of huge files, where more detailed quality of service parameters are needed, is not supported with fine granularity.

\subsection{Comparison of the Approaches}

The presented approaches differ in the areas of usability, granularity of service parameter selection and in terms of changes necessary to the network subsystem of the operating system or to the applications. A summary of the differences is given in figure [5] Especially the possibility to determine the quality of service parameters of the web-server makes a difference. As indicated before, the specification of a parameter set will be useful when downloading huge files or requesting a multimedia stream. On the other hand, this specification is demanding for the user and creates too much overhead in short interactions. Up to now, it is not clear what exact kind of mechanisms supporting short-term reservations will take place in the Differentiated Services Architecture; thus, the tradeoff between usability and more detailed parameter specification can not be decided yet.

\section{The Implementation}

A prototypic implementation using the Linux operating system was done. The current implementation is based on the kernel version 2.2.5. Also changes to the 


\begin{tabular}{|l|c|c|c|}
\hline & $\begin{array}{l}\text { Service Differentiation } \\
\text { via separate HTML Links }\end{array}$ & $\begin{array}{l}\text { Service Differentiation } \\
\text { via HTTP Tags }\end{array}$ & $\begin{array}{l}\text { Service Differentiation } \\
\text { via Packet Marking }\end{array}$ \\
\hline Usability & easy to use & Demanding for the user & easy to use \\
\hline $\begin{array}{l}\text { Granularity of } \\
\text { service selection }\end{array}$ & coarse & fine & very coarse \\
\hline $\begin{array}{l}\text { Changes to } \\
\text { applications }\end{array}$ & web-server & $\begin{array}{c}\text { web-server and web- } \\
\text { browser }\end{array}$ & \\
\hline $\begin{array}{l}\text { Changes to } \\
\text { operating system }\end{array}$ & on server side & on server side & on server and client side \\
\hline
\end{tabular}

Fig. 5. Comparison of the Presented Approaches

web-server had to be made. As for Linux, the Apache web-server is available in source code and runs successfully on thousands of computers. The version number of the Apache web-server was 1.3.9.

The implementation was tested in the UNIQuE testbed described in 6]. The UNIQuE testbed comprises a fully equipped Differentiated Services Domain and several end systems. The realization of Differentiated Services support in the software routers of the testbed is based on the KIDS implementation (cf. [7]).

In the remainder of this section, the elements of the implementation are described. A small set of building blocks is sufficient to realize all described approaches. The modifications of the web-browser, as necessary for the second approach described in section 3.2, have not yet been implemented. The feasibility of this approach seems clear when realizing the first approach given in section 3.1 .

\subsection{Packet Marking}

As indicated by its name, the Packet Marker marks outgoing traffic; i.e. sets the codepoint in the DS field of the IP packet. The input of the packet marker differs between the approaches: Whereas in the first and second approach the desired codepoint is given to the packet marker from the web-server application, in the third approach the packet marker gets this input from the incoming packets. In both cases, already existing structures in the Linux kernel could be used: For each HTTP Get request a new TCP connection is opened and a socket is created. Associated with each socket is a variable called ip_tos that controls the DS field of outgoing IP packets. Packet marking is done via changing the value of ip_tos to the desired codepoint; all IP packets leaving this socket now will get the new ip_tos value in the DS field of the header. Therefore, the modification needed was either to copy the codepoint of the incoming IP packet to this variable or to allow the web-server application to set it. The latter can be done via the system call setsockopt. 


\subsection{DS Profile}

As mentioned before, traffic shaping can already be done in the host. Doing this, allows for fine granular classification and avoids packet discards at the boundary node. When performing shaping of a traffic flow (TCP-traffic in the case of the web-server), the appropriate place is in the transport layer. The implementation is based on shaping sockets, i.e. the shaping of the traffic flows is associated with a socket and done inside the TCP layer of the TCP/IP protocol stack. Therefore, the socket structure was extended with a pointer to a DS profile used by the traffic shaper. The DS profile contains the parameters rate and burst_size which can be set from user-space by an extension of the setsockopt system call. In order to avoid collisions with the current TCA, these values can be checked with the Bandwidth Broker and can be downgraded if necessary.

\subsection{Traffic Shaping}

To shape the traffic according to the desired rate and burst-size, a token bucket is used. The parameters rate and burst_size stored in the DS profile are therefore transformed to token rate and token bucket size. Besides these parameters, the DS profile keeps also internal parameters like the variable last_sent which determines the last send time and a pointer to the delay-queue. The token bucket implemented is a byte-oriented token bucket to achieve higher accuracy. As it works with TCP-packets, a packet-oriented bucket would not be precise enough as their length can vary extremely. Two functions are required for shaping. The first one (ps_shaping () ) is called before we pass the packet from TCP to IP (cf. figure 6). It checks for tokens and either sends the packet immediately or queues it. The second one (delayed_xmit()) processes the delay-queue and is invoked on demand by a timer (cf. figure 7).

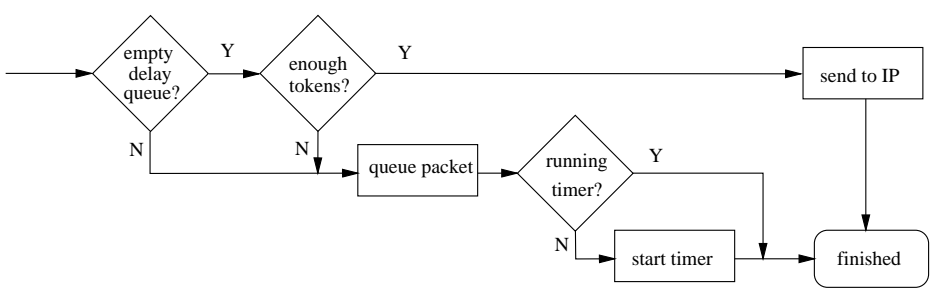

Fig. 6. The Function ps_shaping()

The token bucket algorithm is realized as follows. In ps_shaping () the packet arrival time current_stamp is taken. The amount of new tokens that were theoretically filled into the bucket between last_sent and current_stamp is calculated by

$$
\text { new_tokens }:=(\text { current_stamp - last_sent)/cycles_per_byte, }
$$




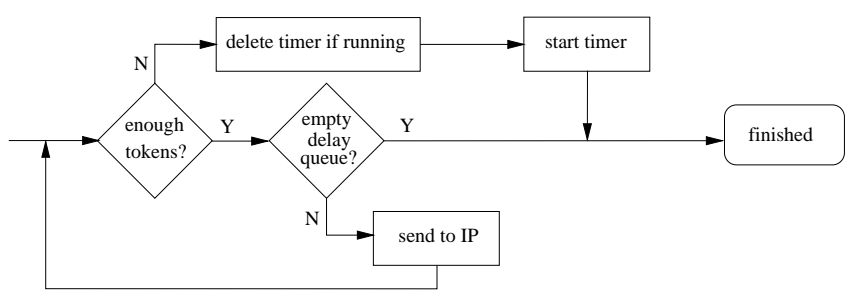

Fig. 7. The Function delayed_xmit

with

$$
\text { cycles_per_byte }:=8 * \text { cpu_clock/rate. }
$$

If now, there are enough tokens to send the packet immediately and the delayqueue is also empty, the amount of tokens in the bucket is updated by adding the new tokens, pruning the tokens against the bucket-size and subtracting the packet-length. last_sent is set to current_stamp. If there are not enough tokens available or the delay-queue is not empty, the packet is enqueued at the end of the delay-queue and a timer for delayed_xmit() is started. Unfortunately, timer in the Linux kernel can only be started with a accuracy of jiffies. Thus, one can not exactly calculate the expiration time $t_{\text {exp }}$ which is calculated by:

$$
\begin{gathered}
t_{\text {exp }}:=(\text { packet-length }-(\text { tokens }+ \text { new_tokens })) / \text { bytes_per_jiffie }, \\
\text { bytes_per_jiffie }:=\text { rate } /(8 * 100)
\end{gathered}
$$

However, a timer is only started if no one is already running. The function delayed_xmit() does almost the same as ps_shaping, except that it processes the delay-queue starting at the head and sends packets as long as there are enough tokens available. If it empties the queue, it stops. If not enough tokens are available, a new timer is started, with the time of expiration calculated as above. Notice, that a running timer is stopped before, as it might not have been calculated on base of the packet at the head of the delay-queue.

\section{Summary}

In this paper three different approaches to enable better services in the World Wide Web were presented. Enabling better services faces the basic problem of the sender orientation of the Differentiated Services Architecture. This conflicts with the typical structure of the client-server interaction in the World Wide Web. The overall quality of service brought to the user at the client side is determined mainly by the quality of service of the response. Therefore the user on the client side must be provided by a means to influence the service level of the server's response. Three approaches to overcome this problem were presented; designed under the constraints of the given Differentiated Services Architecture. These approaches differ in the complexity and location of changes. Also the ease of use for the user differs. As shown, there is a tradeoff between ease of use and an exact determination of parameters by the user. 


\subsection{Future Topics}

The provision of better services in the World Wide Web using the Differentiated Services depends on two factors: The future management architecture of services from end to end is not yet clear, and connected with this, the same can be said about the problem of reservations. The current development of Differentiated Services focuses on more static reservations, but from the beginning on [3], as services would evolve, more dynamic service level agreements were envisioned. Depending on this evolution, the presented approaches might need further adaptation and modification. The sometimes discussed deployment of reservation protocols like RSVP with DiffServ-Networks e.g. would have a strong impact on the provision of end-to-end services in the context of the Differentiated Services Architecture.

\section{References}

1. Baker, F., Heinanen,J., Carlson,M., Davies,E., Wang,Z., Weiss,W.: An Architecture for Differentiated Services. RFC 2475, IETF (1997)

2. Bless, R., Wehrle, K.: Evaluation of Differentiated Services using an implementation under Linux. In: Proceedings of the 7th IFIP Workshop on Quality of Service, IEEE (1999)

3. Nichols, K., Van Jacobson, Zhang, L.: A Two-bit Differentiated Services Architecture for the Internet. RFC2638, IETF (1999)

4. Berners-Lee, T., Masinter, L., McCahill, M.: Uniform Resource Locators (URL). RFC1738, IETF (1994)

5. Bechler, M., Ritter, H., Schiller, J.H.: Quality of Service in Mobile and Wireless Networks: The Need for Proactive and Adaptive Applications. In: Proceedings of the 33rd Annual IEEE Hawai'i International Conference on System Sciences (HICSS), IEEE (2000)

6. Bechler, M., Ritter, H., Schiller, J.H.: Research in the UNIQuE Environment: Mobile Multimedia Services. In: Proceedings of the 3rd IASTED/ISMM Conference on Internet and Multimedia Systems and Applications (IMSA'99), IEEE (1999)

7. Karlsruhe Implementation of Differentiated Services (KIDS) homepage, http://www.telematik.informatik.uni-karlsruhe.de/Forschung/KIDS.html (1999) 